\title{
New Frontiers Titan Orbiter
}

\author{
A 2020 Planetary Science Decadal Survey Whitepaper
}

Jason W. Barnes: University of Idaho, jwbarnes@uidaho.edu (208)310-2079

Alexander G. Hayes (Cornell), Jason M. Soderblom (MIT), Shannon M. MacKenzie (JHU/ APL), Jason D. Hofgartner (JPL/Caltech), Ralph D. Lorenz (JHU/APL), Elizabeth P. Turtle (JHU/APL), Jani Radebaugh (BYU), Devon Burr (NAU), Juan Lora (Yale), Gregory Neumann (NASA GSFC), Steve Vance (JPL/Caltech), Rosaly Lopes (JPL/Caltech), Conor Nixon (NASA GSFC), Paul Corlies (MIT), Leonardo Regoli (UMich), Ella Sciamma-O'Brien (NASA Ames), Rebecca Schindhelm (Ball Aero), Sèbastien Rodriguez (UParis,IPGP), Patrice Coll (UParis), Stèphane Le Mouèlic (UNantes), Michael Heslar (UIdaho), Rajani Dhingra (JPL/Caltech), Jordan Stekloff (PSI), Ed Sittler (NASA GSFC), Anezina Solomonidou (ESA), Michael J. Malaska (JPL/Caltech), Catherine Neish (UWO/PSI), Nicholas Teanby (UBristol), Sandrine Vinatier (ObsParis), Samuel Birch (MIT), Sarah Hörst (JHU/APL), Athena Coustenis (ObsParis), Erich Karkoschka (LPL), Ellen Czaplinski (UArkansas), Paul Hayne (CU), Shawn R. Brueshaber (UWMich), Anthony D. Maue (NAU), Chuanfei Dong (Princeton), Thomas Cornet (ESA), Alfred McEwen (UArizona), Kerry Ramirez (ASU), Emilie Royer (PSI), Farid Salama (NASA Ames), Mark Elowitz (Open U), Shiblee Barua (NASA GSFC, USRA), Miriam Rengel (MPS Germany), Chris McKay (NASA Ames), Nicholas A. Lombardo (Yale), Mathieu Lapôtre (Stanford), Ari Koeppel (NAU), Lori K. Fenton (SETI), Timothy A. Goudge (UT Austin), Morgan L. Cable (JPL/Caltech), Tilmann Denk (DLR Berlin), Tuan H. Vu (JPL/Caltech), Delphine Nna-Mvondo (UMBC/NASA GSFC), Daniel Cordier (CNRS, U Reims), Sebastien Lebonnois (LMD/IPSL, CNRS, Sorbonne U), Andrew Coates (U Coll London), Liliana Lefticariu (SIU), J. Michael Battalio (Yale), Sarah Fagents (U Hawai'i), Paolo Tortora (U Bologna), Ross A. Beyer (SETI), Thomas W. Momary (JPL/Caltech), Mark A. Gurwell (CfA, Harvard, Smithsonian), Lauren Schurmeier (U Hawai'i), Marc Neveu (UMCP/NASA GSFC), Sarah Hörst (JHU), Jennifer Hanley (Lowell), Ajay B. Limaye (UVirginia), Chloé Daudon (UParis/IPGP), Ravindra T. Desai (Imperial College London)

\footnotetext{
Abstract As one of two planetary objects (other than Earth) that have solid surfaces, thick atmospheres, and astrobiological significance, Titan, like Mars, merits ongoing study with multiple spacecraft. We propose that a Titan orbiter dedicated to geophysics, geology, and atmospheric science be added to the New Frontiers menu for the coming decade.
} 


\section{Overview}

We recommend a New-Frontiers-class Titan orbiter to complement Dragonfly with global geology, geophysics, and atmospheric science. Cassini's groundbreaking discoveries on Titan [e.g., 1], in Titan [e.g., 2], and above Titan [3] provide the impetus to ask exciting new questions about the surface, interior, and atmosphere. Titan's well-understood, benign environment allows those questions to be answered with a series of mid-class missions, and does not require Flagship-level exploration.

The New Frontiers Titan Orbiter (NFTO) seeks to answer science questions suggested by Cassini discoveries and similar in scope to those of the orbiter element of previously studied comprehensive Flagships [4, 5]:

1. How does Titan's surface affect its atmospheric circulation, and how does atmospheric circulation drive surface geological and hydrological ${ }^{1}$ processes?

2. What is the extent of Titan's water-ocean mantle, and how does Titan's interior drive surface processes, including tectonism and cryovolcanism?

We envision a science instrument package including at least (but not limited to) fineresolution ( $25 \mathrm{~m} /$ pix or better) surface imaging (NIR camera or radar), altimetry (RADAR or near-infrared laser), weather imaging (wide-field camera), and gravity tracking. The measurements to be made would transform our understanding of Titan in a manner perhaps parallel to that achieved at Mars by Mars Global Surveyor.

Previous Titan Flagship studies, like Titan Explorer [4] and the Titan Saturn System Mission [TSSM; 5], considered three-element missions with an orbiter, lander, and balloon (Figure 1). Given the clever approach of Dragonfly [6] in combining landed and aerial elements into the rotorcraft lander Dragonfly, the opportunity exists to achieve the most important TSSM science with just two medium-class missions. NFTO provides the necessary global scale observations to complement Dragonfly-style local and regional science.

\section{Science Drivers}

Cassini's exploration revealed what lies beneath Titan's haze, and in so doing taught us what questions to ask with follow-on missions. Surely questions about extraterrestrial oceanography [8], evaporite flats [9], and vast sand seas [10] would have seemed farfetched 15 years ago! A medium-class Titan orbiter like NFTO would allow us to answer many of the global physical questions that Cassini has driven us to pose by taking Titan interior, surface, and atmosphere observations to the next level.

Hydrology Titan's global hydrology serves as a prime exemplar of the type of outstanding question that NFTO answers. Huygens observed fluvial channels in the highlands near its landing site [Figure 2; 11]; Cassini observed snippets of individual channels [12] and a fair fraction of a few particularly wide dendritic features [13]. These discoveries revealed that Titan has the only known active hydrological system outside of Earth [14].

But to understand why liquid accumulates where it does [mostly at the north pole, see Figure 3; 15, 16], where sediment gets transported within a comprehensive source-to-sink

\footnotetext{
${ }^{1}$ Although composed of methane, ethane, and dissolved nitrogen, Titan's liquids play a geological role akin to that of water on Earth, hence we use the word "hydrological" in reference to those physical processes and not in reference to chemical composition.
} 

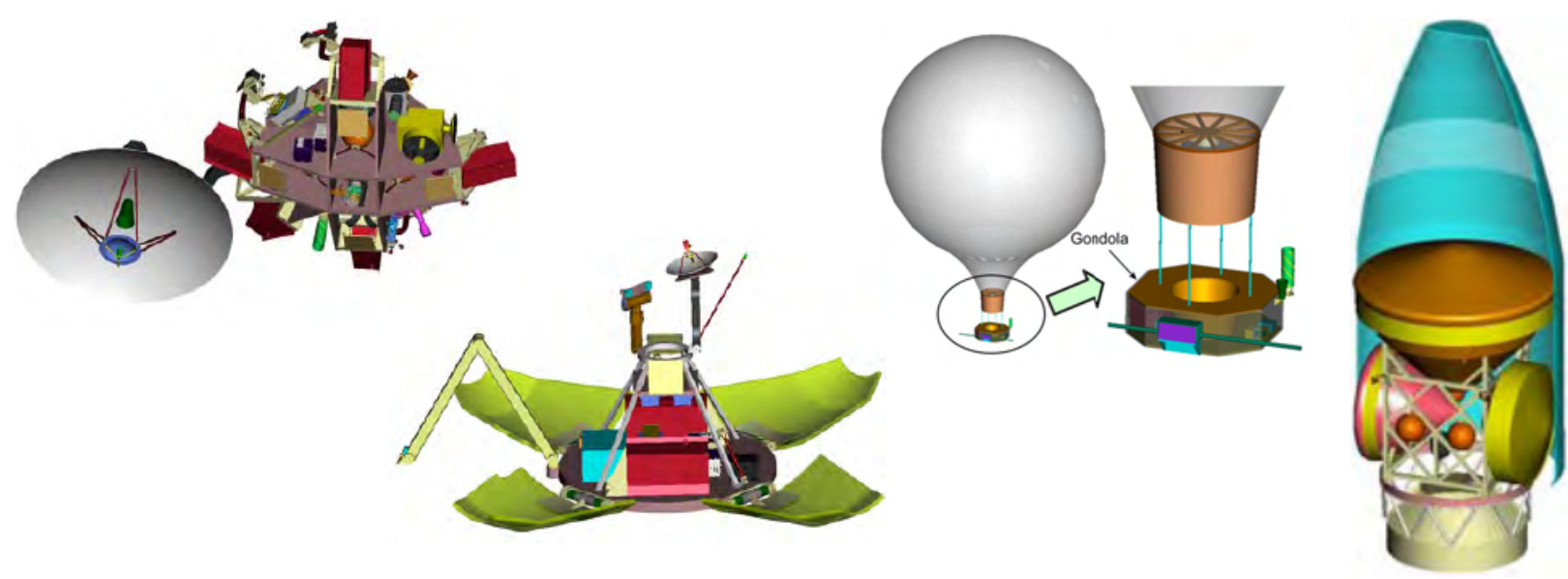

Figure 1: The 2008 Titan Explorer study [4, 7] suggested a three-element Flagship mission: an orbiter, a lander, and a balloon. Although that study envisioned these to be launched together as a single package (right), this ambitious endeavor can also be accomplished over time with a series of more easily digestible medium-class missions. As evidenced by Huygens, Titan's fluvial features are far more extensive than Cassini could resolve.

framework [17, 18], and how methane cycles around the globe [19] requires a follow-up mission. Improving on Cassini in these areas drives us to finer-scale imaging, global imaging and topography coverage, and near-continuous monitoring. NFTO would delineate watersheds [fine-scale imaging, altimetry] [20], map channel networks to investigate the extent and timing of fluvial dissection and sediment transport [fine-scale imaging] [21, 22], diagnose groundwater transport [23], track cloud formation and evolution [wide-field imaging] [24, 25, 26], search for changes indicating rainfall events [wide-field imaging] [27, 28], and ascertain the location and areal extent of all surface liquids using specular reflections [wide-field imaging] [29]. We would then have inputs that we need to advance an integrated atmospheresurface methane transport model [e.g., 30], revealing for the first time the entirety of an extraterrestrial hydrological cycle.

Dunes Cassini resolved dunes [31] and interdunes [32]. Titan's sand dunes resemble those found on Earth in morphology (their longitudinal type matches those in the Saharan, Arabian, and Namib deserts) and in the compositional diversity of sand-free interdunes. Most large longitudinal dunes on Earth, however, are compound in form, showing different smaller dune types superimposed on the larger structure as resulting from shorter-term climatic shifts $[33,34]$. To take the next steps from Cassini's coarse-resolution regional observations, NFTO would be able to identify and characterize these smaller structures across all of Titan's sand seas [fine-scale imaging], thus pointing to the stability of Titan's climate over the past millions of years. A Titan orbiter would also place the dunes into global context [35], ascertaining sand transport patterns, sources, and sinks [fine-scale imaging, altimetry].

Cryovolcanoes While cryovolcanoes have been suggested to be the ultimate source for replenishing Titan's leaking methane [37], definitive identification of cryovolcanic edifices on the surface has not been possible with Cassini data [38, 39]. Thus, the ultimate source of Titan's atmospheric methane remains unknown. NFTO would execute a global search for 


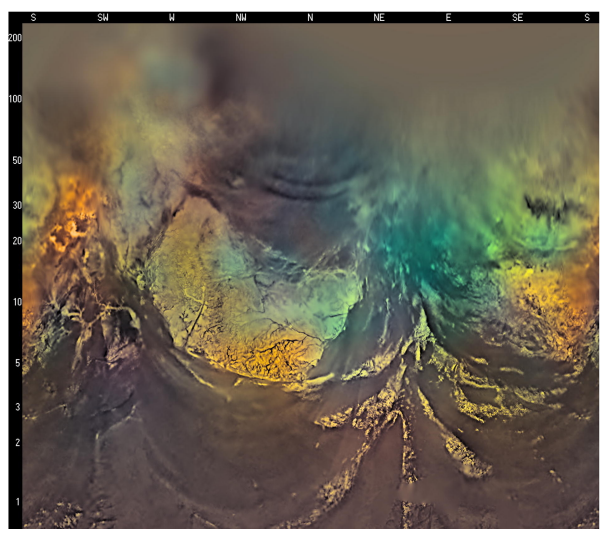

Figure 2: Huygens' view of channel-dissected highlands near its landing site [36] provides a tantalizing hint of what NFTO's global fine-resolution imaging would reveal. The vertical axis labels at left refer to distance from the landing site in $\mathrm{km}$, while the cardinal directions at top show azimuthal directions. The river valleys shown here are $20-50 \mathrm{~m}$ wide.

new candidates as well as evaluate the merit of current ones like Doom Mons [40] - fineresolution stereo in particular holds promise for placing morphologic structures identified by imaging into local topographic context. Evidence for flow features would suggest Titan's ocean is in active communication with its organic-rich surface. That interaction could be why Titan has an atmosphere, while its sister icy satellites have none.

Layering Ultimately we can determine whether or not Titan functions as a sedimentary world by imaging the walls of canyons [41], labyrinths [42], crater rims [43], mountains [44], and shorelines [23] to read Titan's geologic history through its stratigraphy. At Mars, discovery of cross-bedded stratigraphy within the walls of Victoria crater allowed Opportunity to read the aeolian history [45]. Observations of Mars' North Polar Layered Deposits show evidence of Milankovic cycles of climate forcing [46]. At a much larger scale, NFTO could look back into Titan's history to constrain long-term climate and sea-level variations and evaluate the duration over which Titan's present thick atmosphere, rainfall, and lacustrine processes have persisted $[47,48]$.

Tectonism Linear mountain chains slice across Titan, but, unlike the vast extensional cracks on Titan's icy satellite siblings, the origins of these features are unknown [49]. The mountains' global patterns are consistent with both extension and compression in the case where the polar lithosphere is thinner than that at the equator [50]. Fine-resolution imaging of mountains and their environs can reveal the dominant stress regime within Titan's crust [fine-scale imaging, altimetry] by revealing the surface expression of faults and tracing their strike and dip [51]. Once we map Titan's full global topography, including its mountain ranges, we can better understand how mountains and topography affect weather and climate.

Subsurface Ocean The liquid water mantle beneath its crust places Titan in the family of Ocean Worlds [52]. The overall thickness of Titan's crust along with the depth of the ocean itself have not been substantively constrained by Cassini $[53,54,55,56]$. Following up on Cassini's multiple flybys, a Titan orbiter could place sufficiently tight constraints on moment of inertia, gravitational moments, and tidal Love numbers [gravity, fine-scale imaging, altimetery] to not only measure the depth to the ocean, but also potentially probe the base of the ocean to determine whether it is in contact with silicate or high-pressure ice [57]. These same measurements could potentially constrain the nature of the ocean as well, such as its density or whether or not it is stably stratified [58]. 


\section{Instruments}

While the Titan Explorer study envisioned an orbiter with 14 instruments, we suggest a threshold set of 4 science experiments: high-resolution surface imaging, global altimetry, weather and change-detection monitoring, and radio science for spacecraft tracking. An advantage of a New-Frontiers-class mission scope is the ability to choose instruments selectively - to skim the scientific cream from the top of the questions outstanding after Cassini.

Surface Imaging Cassini's imaging with RADAR, VIMS, and ISS transformed Titan from an orange ball into a diverse, active, and exciting world. Discovery and mapping of sand dunes [31], thunderstorms [minus the thunder; 59], seas [15, 60], dust storms [61], evaporites [62], and rainfall-induced surface changes [63], among many other meteorologic, geologic, and geophysical phenomena, show Titan's surface-atmosphere interactions to be second only, if not similar, to Earth's in complexity. True surface resolution of RADAR and the best VIMS and ISS imaging was $\sim 1 \mathrm{~km}$ (surface sampling could be as fine as $250 \mathrm{~m} /$ pixel [32], but speckle noise and low SNR hampered Nyquist-sampled actual resolution), and at surface sampling $<350 \mathrm{~m} /$ pixel RADAR only covered $20 \%$ of the surface.

NFTO would achieve global ( $>90 \%$ coverage) coverage at $10 \mathrm{x}$ better resolution than Cassini - finer than $100 \mathrm{~m}$ resolution using $25 \mathrm{~m}$ or better pixel scale [JETwavelength]. Overlapping images would allow generation of local Digital Elevation Models with vertical accuracy down to tens of meters. Global imaging would reveal changes in Titan's dynamic surface since the Cassini mission. The imaging could in principle be acquired by either a synthetic aperture radar or a near-infrared imager [JETwavelength].

Weather \& Surface Change Imaging Titan's dynamic atmosphere exhibits variations in cloud cover [64], haze [65], and even occasional dust storms [61]. These atmospheric phenomena lead to changes in the appearance of Titan's surface on the timescale of weeks $[27,66,28]$. To constrain weather processes, NFTO would measure cloud evolution, track cloud movement, and observe surface changes using a wide-angle near-infrared camera.

The wide-angle weather camera would observe Titan's dayside both on repeated adjacent orbits and over the course of single orbits. It would use three different near-infrared colors to provide altitude discrimination and constrain reflective properties. This camera would also

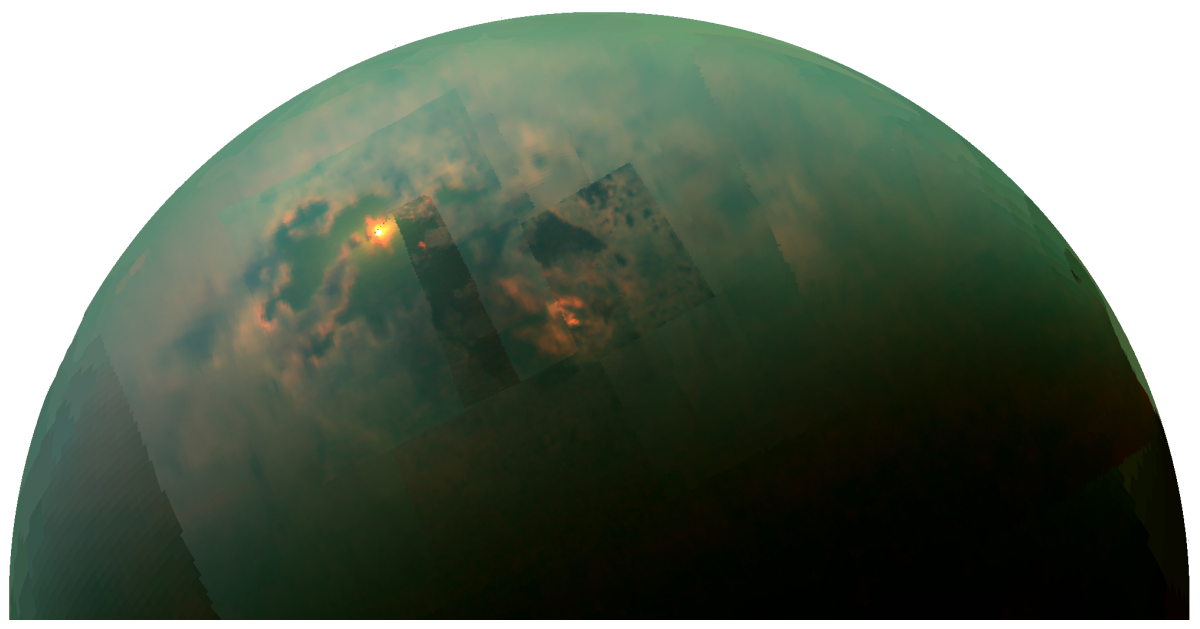

Figure 3: Cassini's view of Titan's north pole showing seas, clouds, landforms, and a superbright specular reflection of the Sun. 
be ideal for using specular reflections [67] to constrain the extent, variation, and roughness [waves; see 68] of liquids covering Titan's surface.

Altimetry Our experience studying planetary geology (in particular the Earth and Mars) has made clear that global topography plays a key role in driving surface geology (particularly hydrology). Present knowledge of Titan's topography is inadequate to understand those surface processes (Figure 4). Accurate global surface topography would reveal the extent of watersheds [20], the nature of mountain-building [44], crater relaxation [43], the vigor of surface runoff and flows [17], the effects of topography on atmospheric circulation [69], the strength of surface materials and their susceptibility to erosion [41], and the role of subsurface liquid methane flow [22]. We expect that global topographic maps will transform our understanding of Titan in the same way that they did our understanding of Mars [70].

Repeat accurate orbital altimetry of the same areas at different orbital phase can also directly measure the deformation of the crust from internal convection and tidal forces, revealing the level of activity in the interior. Determination of tidal Love numbers from altimetry and gravity will place strong constraints on Titan's interior structure [71, 72]. Altimetric and imaging observations of Titan's seas over the course of its orbit around Saturn would allow us to directly measure the level of tidal sloshing within and between methane seas.

Gravity The NFTO gravity investigation would employ an ultrastable oscillator coupled with its radio transmitter to accurately measure the spacecraft's acceleration, using Titan's gravity field to probe its interior $[73,74,55]$. Cassini's flybys constrained Titan's gravity field to degree 5 [2]; spending a much greater fraction of its time near to Titan, and moving at a much slower velocity, a Titan orbiter would provide a more comprehensive view. These higher order harmonics can distinguish between models of crustal thickness variation Pratt vs. Airy isostasy with respect to Titan's equator-to-pole tophographic variations [75]. When combined with tidal measurements [58], gravity provides a method for peering into Titan's interior, where it could reveal the vertical extent of Titan's water ocean and whether or not its lower boundary abuts a silicate mantle or a layer of high-pressure ice [albeit with potential model dependence; 76]. A thin crust would facilitate surface-ocean interaction.

Gravity observations might also reveal whether Titan's interior exerts active tectonic stresses on the lithosphere, building and supporting mountains, causing extension, or possibly forcing cryovolcanic liquids to the surface.

\section{Strategic Value}

The previous Decadal Survey, Vision and Voyages [79], did not consider a standalone Titan orbiter because "it was not proposed by community white papers, and because of the advantages of an integrated orbiter and in situ elements both for delivery to Saturn and for data relay" [79]. The Flagship mission that Vision and Voyages did study, TSSM [5], was judged to have science "on a level equivalent to the science of the Europa Jupiter System Mission", but was nevertheless "deferred to the decade following 2013-2022 primarily because of the greater technical readiness of JEO". Vision and Voyages went on to say that "[i]ts high scientific priority, however, is especially noteworthy. Because the Titan Saturn System 


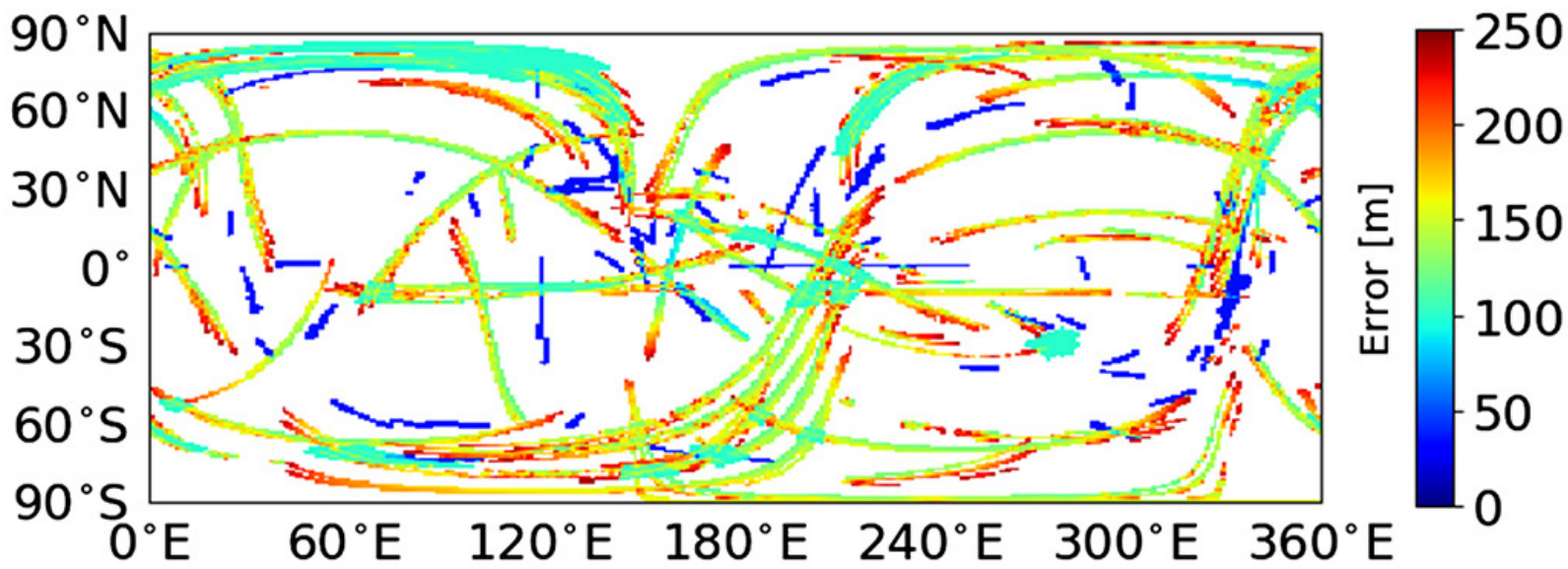

Figure 4: Our current best estimate of Titan's global topography, from the Cassini RADAR, remains a haphazard and incomplete map. This image shows the location of existing Titan topography data and their accuracy [77, 78]. The most accurate topography, from RADAR altimetry, is available as just a few dozen widely spaced one-dimensional strips evident by dark blue in this figure.

Mission is a particularly strong candidate for the future, continued thorough study of it is recommended" (emphasis in original).

Given Titan's high priority and the selection of the Dragonfly lander, the opportunity exists in the coming decade to complete the scientific scope of a Flagship mission with a standalone New Frontiers Titan Orbiter. Such a mission requires no new technology developments. Titan's complexity and astrobiological potential rank it along with Mars as the highest priority targets in the Solar System for continued, consistent exploration with multiple ongoing, parallel missions. The compelling science of NFTO complements and extends Dragonfly without conflicting, and thus the Decadal Survey should recommend that a mid-class Titan mission be flown, or at least added to the New Frontiers menu, in the coming decade.

1. A. G. Hayes, Annual Review of Earth and Planetary Sciences 44, 57-83 (June 2016).

2. D. Durante et al., Icarus 326, 123-132 (July 2019). 3. I. Müller-Wodarg et al., Titan (Cambridge, Mar. 2014). 4. R. D. Lorenz et al., presented at the Space Technology and Applications International Forum-STAIF 2008, ed. by M. S. El-Genk, vol. 969, pp. 380-387.

5. K. Reh et al., JPL Task Order \#NMO710851 (2009). 6. E. P. Turtle et al., presented at the Lunar and Planetary Science Conference, vol. 49, p. 1641. 7. J. C. Leary et al., JHU/APL Public Release Version (2008). 8. R. D. Lorenz et al., Icarus 237, 9-15 (July 2014). 9. S. M. MacKenzie et al., ApJ 821, 17 (Apr. 2016). 10. J. Radebaugh et al., Icarus 194, 690-703 (Apr. 2008). 11. L. A. Soderblom et al., Planet. Space Sci. 55, 2015-2024 (Nov. 2007). 12. C. Elachi et al., Nature 441, 709-713 (June 2006). 13. J. W. Barnes et al., J. Geophys. Res.(Planets) 112, E11006 (Nov. 2007). 14. A. G. Hayes et al., Nature Geoscience 11, 306-313 (May 2018). 15. E. R. Stofan et al., Nature 445, 61-64 (Jan. 2007). 16. O. Aharonson et al., Nature Geoscience 2, 851-854 (Dec. 2009). 17. D. M. Burr et 
al., Icarus 181, 235-242 (Mar. 2006). 18. J. Grotzinger et al., Comparative Climatology of Terrestrial Planets 1, 439-472 (2013). 19. J. L. Mitchell et al., Annual Review of Earth and Planetary Sciences 44, 353-380 (June 2016). 20. R. D. Dhingra et al., Icarus 299, 331-338 (Jan. 2018). 21. D. M. Burr et al., Geological Society of America Bulletin 125, 299-321 (2013). 22. A. G. Hayes et al., GRL 44, 11 (Dec. 2017). 23. J. W. Barnes et al., Icarus 201, 217-225 (May 2009). 24. C. A. Griffith et al., Science 313, 1620-1622 (Sept. 2006). 25. S. Rodriguez et al., Icarus 216, 89-110 (Nov. 2011). 26. E. P. Turtle et al., GRL 45, 5320-5328 (2018). 27. E. P. Turtle et al., GRL 36, L2204 (Jan. 2009). 28. R. D. Dhingra et al., GRL 46, 1205-1212 (Feb. 2019). 29. J. M. Soderblom et al., presented at the AAS/DPS \#49, vol. 49, p. 213.08. 30. S. P. Faulk et al., Nature Astronomy 4, 390-398 (Dec. 2019). 31. R. D. Lorenz et al., Science 312, 724-727 (May 2006). 32. J. W. Barnes et al., Icarus 195, 400-414, doi:10.1016/j.icarus.2007.12.006 (May 2008). 33. N. Lancaster, The Geomorphology of Desert Dunes (Routledge, 1995). 34. R. C. Ewing et al., Nature Geoscience 8, 15-19 (Jan. 2015). 35. J. Radebaugh et al., Geomorphology 121, Planetary Dune Systems, 122 -132 (2010). 36. E. Karkoschka et al., Icarus 270, 307-325 (May 2016). 37. C. Sotin et al., Nature 435, 786-789 (June 2005). 38. R. M. C. Lopes et al., Icarus 186, 395-412 (Feb. 2007). 39. J. M. Moore et al., Icarus 212, 790-806 (Apr. 2011). 40. R. M. C. Lopes et al., J. Geophys. Res.(Planets) 118, 416-435 (Mar. 2013). 41. V. Poggiali et al., GRL 43, 7887-7894 (Aug. 2016). 42. M. J. Malaska et al., Icarus, 113764 (2020). 43. C. D. Neish et al., Icarus 223, 82-90 (Mar. 2013). 44. J. Radebaugh et al., Icarus 192, 77-91 (Dec. 2007). 45. A. G. Hayes et al., J. Geophys. Res.(Planets) 116, E00F21 (Apr. 2011). 46. P. Becerra et al., GRL 44, 62-70 (Jan. 2017). 47. G. Tobie et al., Nature 440, 61-64 (Mar. 2006). 48. P. Becerra et al., J. Geophys. Res.(Planets) 121, 1445-1471 (Aug. 2016). 49. G. Mitri et al., J. Geophys. Res.(Planets) 115, E10002 (Oct. 2010). 50. C. Cook-Hallett et al., J. Geophys. Res.(Planets) 120, 1220-1236 (June 2015). 51. Z. Y.-C. Liu et al., Icarus 270, 14-29 (May 2016). 52. F. Nimmo et al., J. Geophys. Res.(Planets) 121, 1378-1399 (Aug. 2016). 53. F. Nimmo et al., Icarus 208, 896-904 (Aug. 2010). 54. D. Hemingway et al., Nature 500, 550-552 (Aug. 2013). 55. G. Mitri et al., Icarus 236, 169-177 (July 2014). 56. R.-M. Baland et al., Icarus 237, 29-41 (July 2014). 57. A. Levi et al., ApJ 882, 71 (Sept. 2019). 58. J. Luan, arXiv e-prints, arXiv: 1905.03802 [astro-ph.EP] (May 2019). 59. C. A. Griffith et al., Science 310, 474-477 (Oct. 2005). 60. A. Hayes et al., GRL 35, L9204 (May 2008). 61. S Rodriguez et al., Nature Geoscience 11, 727 (2018). 62. J. W. Barnes et al., Icarus 216, 136-140 (Nov. 2011). 63. E. P. Turtle et al., Science 331, 1414-1417 (Mar. 2011). 64. M. E. Brown et al., Icarus 205, 571-580 (Feb. 2010). 65. C. Sotin et al., presented at the AAS/DPS \#48, vol. 48, p. 515.08. 66. J. W. Barnes et al., Planetary Science 2:1 (2013). 67. K. Stephan et al., GRL 37, L7104 (Apr. 2010). 68. J. W. Barnes et al., Planetary Science 3 (2014). 69. S. M. MacKenzie et al., Journal of Geophysical Research (Planets) 124, 1728-1742 (July 2019). 70. D. E. Smith et al., Science 284, 1495 (May 1999). 71. J. M. Wahr et al., J. Geophys. Res.(Planets) 111, E12005 (Dec. 2006). 72. A. Genova et al., Icarus 272, 228-245 (July 2016). 73. L. Iess et al., Science 327, 1367-(Mar. 2010). 74. L. Iess et al., Science 337, 457 (July 2012). 75. C. A. Nixon et al., Planet. Space Sci. 155, 50-72 (June 2018). 76. S. D. Vance et al., J. Geophys. Res.(Planets) 123, 180-205 (Jan. 2018). 77. P. Corlies et al., GRL 44, 11 (Dec. 2017). 78. R. D. Lorenz et al., Icarus 225, 367-377 (July 2013). 79. S. Squyres et al., National Academies Press, Washington, D.C. (2011). 